\title{
Model of Arterial Tree and Peripheral Control for the Study of Physiological and Assisted Circulation
}

\author{
E Lanzarone, P Liani, G Baselli, ML Costantino
}

Department of Bioengineering, Politecnico di Milano, Milan, Italy

\begin{abstract}
Peripheral vasomotion, interstitial liquid exchange and cardiovascular system behaviour during extra corporeal circulation are investigated by means of a lumped parameter model of the systemic arterial circulation, made by 63 RCL segments and 30 peripheral districts.

A considerable autoregulatory activity of peripheral districts was found: peripheral blood flow keeps constant over a wide pressure range and, when regulated, oscillates around its physiological value.

Then parameters alterations deriving from extra corporeal circulation are considered, observing peripheral and large vessel response.
\end{abstract}

\section{Introduction}

This work consists in the development of a lumped parameter model of arterial fluid dynamics, constituted by large artery segments and peripheral districts, and the implementation of the local regulation mechanisms on the model itself.

These mechanisms are implemented in the peripheral districts: myogenic control of the arterioles, depending on arteriolar blood pressure; metabolic control of the venules, depending on venous blood oxygen concentration; and mechanical effects of the interstitial pressure variation, due to the exchange of liquid through the capillary membrane, on the capillaries.

The effects both of an imposed pressure entering peripheral districts, to evaluate autoregulatory oscillatory behaviour, and of extra corporeal circulation ECC conditions on the whole system were studied.

\section{Methods}

The model considers the arterial tree from the aortic valve to the veins entrance. It is constituted of 63 large artery segments and 30 peripheral networks (Fig.1).

The input is represented by the blood flow through the aortic valve, which in physiological conditions is in terms of the Swanson and Clark expression [1].

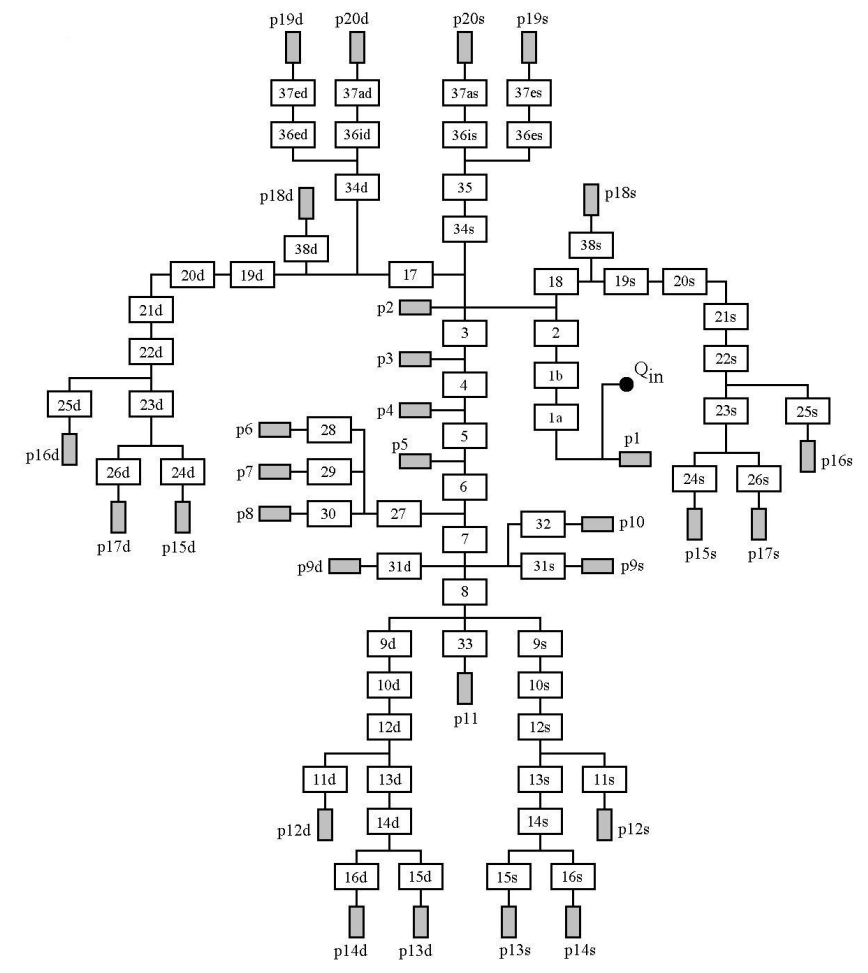

Fig.1: Arterial tree: large artery segments are indicated by a number sometimes followed by letters and peripheral network by the letter $\mathrm{p}$ followed by numbers and letters.

\subsection{Large artery segments}

Each segment was modelled with an electrical equivalent, characterized by:

- viscous Hagen-Poiseuille resistance and inertance for blood motion:

$$
\mathrm{R}=\frac{8 \cdot \mu \cdot 1}{\pi \cdot \mathrm{r}^{4}} \quad \mathrm{~L}=\frac{\rho \cdot 1}{\pi \cdot \mathrm{r}^{2}}
$$

- compliance and dissipative resistance for viscoelastic wall behaviour [2]:

$$
\mathrm{C}=2 \frac{\pi \cdot 1 \cdot \mathrm{r}^{3}}{\mathrm{E} \cdot \mathrm{s}} \mathrm{C} \cdot \mathrm{R}_{\mathrm{v}}=0,002 \mathrm{sec}
$$

Geometrical and mechanical parameters are evaluated from other literature works [3], while blood volume mass 
and viscosity are a function of temperature and hematocrit.

The configuration of the segment is a $\pi$ one with the dissipative resistance of wall in series to the compliance.

\subsection{Peripheral networks}

Peripheral networks are also implemented by means of an electrical analogy, in which every resistance represents a number $\mathrm{N}$ of vessels in parallel (Fig.2).

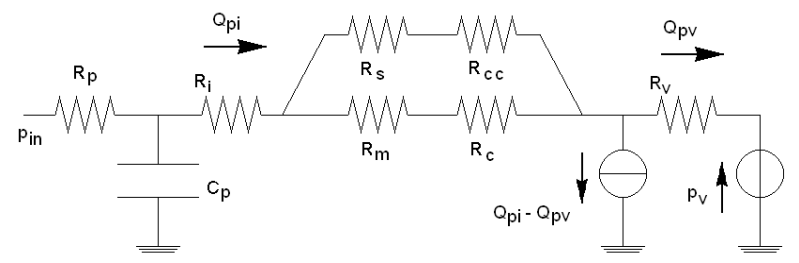

Fig.2: Peripheral network.

Different kinds of vessel can be distinguished: terminal arteries $R_{p}$, large arterioles $R_{i}$, small arterioles $R_{m}$ and $R_{s}$, capillaries $R_{c}$ and $R_{c c}$, venules $R_{v}$. Each one is characterized by the same geometrical dimensions in each peripheral network. Peripheral networks are equal except for the number of vessels and the compliance $\mathrm{C}_{\mathrm{p}}$. The venous pressure generator $\mathrm{p}_{\mathrm{v}}$ assumes a value of $8 \mathrm{mmHg}$.

The flow $Q_{p v}$ in venules is different from the one entering the district, due to the filtration effects trough capillary membrane, described by the Starling law:

$$
\mathrm{J}_{\text {solvent }}=\mathrm{L}_{\mathrm{p}} \cdot(\Delta \mathrm{p}-\Delta \pi)
$$

where $\mathrm{L}_{\mathrm{p}}$ is the permeability of the membrane [4] and $\Delta \mathrm{p}$ e $\Delta \pi$ the hydrostatic and oncotic pressure difference between inside and outside of the vessel.

The capillary hydrostatic pressure is expressed as a linear drop on the capillary length form $\mathrm{p}_{\text {in }}$ to $\mathrm{p}_{\text {out }}$.

The capillary oncotic one is expressed as a function of the proteinic concentration, according to Landis [5]:

$$
\pi_{\text {int }}=\mathrm{a}_{1} \cdot \mathrm{c}_{\mathrm{int}}+\mathrm{a}_{2} \cdot \mathrm{c}_{\mathrm{int}}{ }^{2}
$$

To allow an analytical integration of the law, proteinic concentration is assumed to be constant and equal to the entrance value $(7.5 \mathrm{~g} / 100 \mathrm{ml}$ in reference conditions).

The flow $\mathrm{Q}_{\mathrm{pv}}$ was obtained integrating along the capillary length and multiplying by the number $\mathrm{N}$ of vessels:

$$
\begin{aligned}
& \mathrm{Q}_{\mathrm{pv}}=\mathrm{Q}_{\mathrm{pi}}-\mathrm{L}_{\mathrm{p}} \cdot 2 \pi \cdot \mathrm{r} \cdot \mathrm{L} . \\
& \cdot\left[\frac{\mathrm{N}_{\mathrm{c}} \cdot \mathrm{p}_{\text {inm }}+\mathrm{N}_{\mathrm{cc}} \cdot \mathrm{p}_{\mathrm{ins}}}{2}+\left(\mathrm{N}_{\mathrm{c}}+\mathrm{N}_{\mathrm{cc}}\right) \cdot\left(\frac{\mathrm{p}_{\text {out }}}{2}-\mathrm{p}_{\text {ext }}-\bar{\pi}_{\text {int }}+\pi_{\text {ext }}\right)\right]
\end{aligned}
$$

\subsection{Metabolic control}

Metabolic control guarantees a sufficient blood flow for oxygen supply to each district, minimizing heart work. It can also adjust the flow according to the variations of metabolic peripheral activity.

It acts on venules on the basis of Groebe's work [6].

The trigger variable is the specific oxygen venous concentration $\mathrm{C}_{\mathrm{V}} \mathrm{O}_{2}$ in the network, flowing through $\mathrm{R}_{\mathrm{v}}$ :

$$
\mathrm{C}_{\mathrm{v}_{2}}=\mathrm{C}_{\mathrm{A}_{2}} \mathrm{O}_{2}-\frac{\text { ConsP }}{\mathrm{Q}_{\mathrm{pi}}}
$$

1. ConsP is the total oxygen consumption of the district in physiological reference conditions, constant and depending on district metabolic activity.

2. $\mathrm{Q}_{\mathrm{pi}}$ is the total flow entering the peripheral network.

3. $\mathrm{C}_{\mathrm{A}} \mathrm{O}_{2}$ is the oxygen concentration in the arterial blood entering the district. It is the same in each peripheral network and it depends on the partial pressure of oxygen, carbon dioxide and on hematocrit, according to the dissociation curve described by Singh and Sharan [7].

First of all, a first order dynamics was introduced in the oxygen concentration before acting on venular radius simulating the presence of chemical mediators with a non-instantaneous response.

$$
\frac{\mathrm{dC}_{\mathrm{r}}}{\mathrm{dt}}=\frac{-\mathrm{C}_{\mathrm{r}}+\mathrm{C}_{\mathrm{V}_{2}}}{10}
$$

Furthermore, the small high-frequency oscillations created inside a cardiac cycle are reduced.

If the filtered concentration is too low the district is in high need of oxygen and the venules dilate and vice versa. The venular radius response was described with a first-order linear system:

$$
\frac{\mathrm{dr}_{\mathrm{v}}}{\mathrm{dt}}=\frac{-\mathrm{r}_{\mathrm{v}}+\mathrm{r}_{\mathrm{vI}}-f\left(\mathrm{C}_{\mathrm{r}}-\mathrm{C}_{\mathrm{v}_{21}}\right)}{\tau_{\mathrm{rp}}} ; f=\text { Sat } \cdot\left(1-\frac{2}{1+\mathrm{e}^{\frac{2 \mathrm{G}}{\mathrm{Sat}}\left(\mathrm{c}_{\mathrm{r}}-\mathrm{C}_{\mathrm{v}} \mathrm{O}_{21}\right)}}\right)
$$

where symbols with the index I represent the reference values. Finally, a pure delay of 2 seconds was introduced in the response of the venules radius.

\subsection{Myogenic control}

Myogenic control has a vasoconstrictive action on the arterioles that increases with the mean pressure of the flowing blood (between the inlet and the outlet). The response is the sum of a passive (dilatation with the increasing of pressure) and an active component, indicating the vasoconstrictive action.

Radius behavior is described according to the experimental studies of Davis [8]:

- diameter passive component:

$$
\mathrm{d}_{\text {pass } \infty}=\mathrm{d}+\mathrm{G} \cdot \mathrm{p}+\mathrm{k} \cdot\left(1-\mathrm{e}^{\mathrm{h} \cdot \mathrm{p}}\right)
$$

- diameter behaviour:

$$
\mathrm{d}_{\infty}=\mathrm{d}_{\text {pass } \infty} \cdot f r ; f r=f r_{\mathrm{A}}+\frac{f r_{\mathrm{B}}}{1+\mathrm{e}^{\frac{\mathrm{p}-\mathrm{p}_{0}}{\tau}}}
$$


- diameter active component (difference):

$$
\mathrm{d}_{\text {att } \infty}=\mathrm{d}_{\infty}-\mathrm{d}_{\text {pass } \infty}
$$

The dynamics describing how the radius behaviour is reached is introduced as a first-order dynamics:

$$
\frac{\mathrm{dr}_{\text {pass }}}{\mathrm{dt}}=\frac{-\mathrm{r}_{\text {pass }}+\mathrm{r}_{\text {pass } \infty}}{\mathrm{T}_{\text {pass }}} ; \frac{\mathrm{dr}_{\text {att }}}{\mathrm{dt}}=\frac{-\mathrm{r}_{\text {att }}+\mathrm{r}_{\text {att } \infty}}{\mathrm{T}_{\text {att }}}
$$

The active component is delayed using a pure delay:

$$
\mathrm{r}_{\text {att }}{ }^{*}(\mathrm{t})=\mathrm{r}_{\text {att }}(\mathrm{t}-\vartheta)
$$

The resulting expression for the radius is the sum of the two contributions:

$$
\mathrm{r}=\mathrm{r}_{\text {pass }}+\mathrm{r}_{\text {att }}{ }^{*}
$$

\subsection{Filtration effects}

When interstitial hydrostatic pressure is different from its reference value of $-6.9 \mathrm{mmHg}$, capillary radius changes:

$$
\frac{\mathrm{dr}}{\mathrm{dt}}=\frac{-\mathrm{r}_{\mathrm{c}}+4 \cdot\left[1-\mathrm{K}\left(\mathrm{p}_{\mathrm{ext}}+6,9\right)\right]}{\tau}
$$

Interstitial pressure directly depends on the interstitial accumulation of liquid:

$$
p_{\text {ext }}= \begin{cases}0,07 \cdot \text { Acc }-6,9 & \text { for Acc }<100 \mathrm{ml} \\ 0,0007 \cdot \text { Acc }-0,069 & \text { for Acc } \geq 100 \mathrm{ml}\end{cases}
$$

Considering the transcapillary flow rate and the effect of the lymphatic system, the behavior of the interstitial accumulation is:

$$
\frac{\mathrm{d} A c c}{\mathrm{dt}}= \begin{cases}\mathrm{Q}_{\mathrm{pi}}-\mathrm{Q}_{\mathrm{pv}}-\text { Lymp } & \text { Acc }>0 \\ \mathrm{Q}_{\mathrm{pi}}-\mathrm{Q}_{\mathrm{pv}} & \text { Acc }<0 \\ 0 & \text { Acc }=0, \mathrm{Q}_{\mathrm{pi}}-\mathrm{Q}_{\mathrm{pv}}<\text { Lymp } \\ \mathrm{Q}_{\mathrm{pi}}-\mathrm{Q}_{\mathrm{pv}}-\text { Lymp } & \text { Acc }=0, \mathrm{Q}_{\mathrm{pi}}-\mathrm{Q}_{\mathrm{pv}} \geq \text { Lymp }\end{cases}
$$

The maximum capacity of the lymphatic system Lymp depends on the pressure of the interstitial fluid:

$$
\text { Lymp }=1,625 \mathrm{ml} / \mathrm{min}-\frac{1,557 \mathrm{ml} / \mathrm{min}}{1+\mathrm{e}^{\frac{p_{\text {ext }}+0,5}{0,719}}}
$$

Finally, even the oncotic pressure of the interstice, whose reference value is $4.5 \mathrm{mmHg}$, is assumed to be a function of the content of the interstitial liquids.

It was obtained from the proteinic mass conservation, and under the hypothesis of a linearity between concentration and pressure:

$$
\pi_{\mathrm{ext}}=\pi_{\mathrm{ext} 0} \cdot \frac{1}{1+\frac{\mathrm{Acc}}{\mathrm{V}_{0}}}
$$

where $\mathrm{V}_{0}$ represents the volume of the liquid present in the interstice in physiological conditions, which is different in each network.

\section{Results}

\subsection{Physiological conditions}

First of all the model behaviour in physiological conditions (temperature $37 \mathrm{C}$, hematocrit $45 \%$, mean flow $5 \mathrm{l} / \mathrm{min}$, heart frequency $75 \mathrm{bpm}$ ) and without regulation mechanisms was verified.

Simulation outcomes are comparable to the other models in literature both in their mean value and trends.

In periphery an attenuation of flow pulsation form terminal arteries to venules is observed (Fig.3).

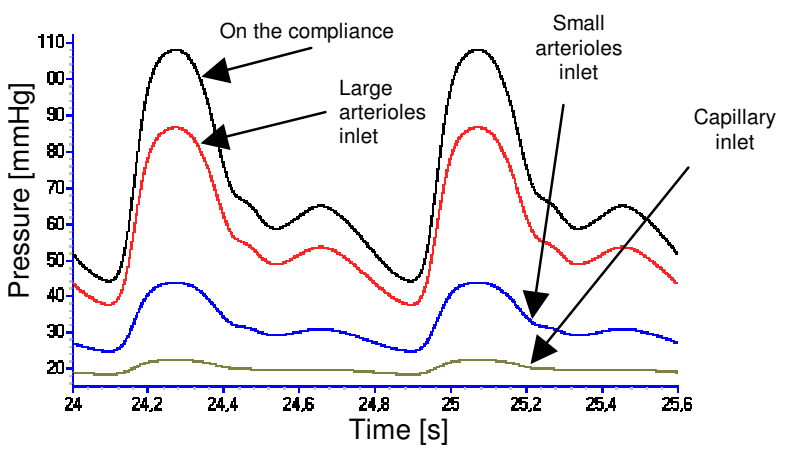

Fig.3: Pressure reference trend in peripheral network.

Control behaviour was studied imposing the inlet pressure of a peripheral district; using a pressure ramp an autoregulatory behaviour was observed (Fig.4).

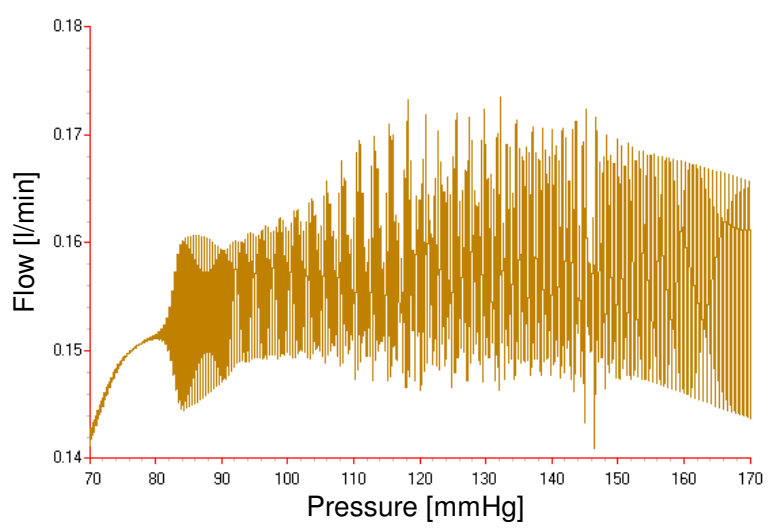

Fig.4: Peripheral flow trend.

In a wide range of imposed pressure $(80-170 \mathrm{mmHg})$ flow assumes a value near to the reference one without control mechanisms and it presents self sustained oscillations.

Working on two peripheral network after a bifurcation, a pressure step entering the segment $14 \mathrm{~s}$ till the mean reference value was imposed. Oscillatory response frequency is variable from network to network and there is an alternance of synchronization and desynchronization periods in total flow; so an oscillations attenuation in the total input flow of the RCL segment was observed (Fig.5). 


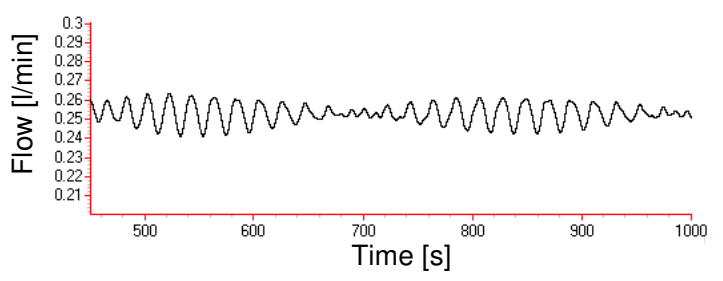

Fig.5: Total bifurcation flow.

\subsection{Extra corporeal circulation conditions}

The whole model was applied to the conditions generated during the ECC: a constant aortic input pressure and consequently a continuous flow was imposed, then only a parameter with a constant input pressure was altered; finally ECC with all the alterations at once was simulated.

1. The continuous flow causes a venous oxygen concentration increase, due to the $\mathrm{E}\left[1 / \mathrm{Q}_{\mathrm{pi}}\right]$ minimum with continuous flow. With the ECC input pressure of $70 \mathrm{mmHg}$ (lower than the physiological one) there is a venular dilatation, due to flow reduction, but limited by venular constriction, due to the continuous flow.

2. With the fall in blood temperature due to hypothermia, the total resistance increases, for the RCL segment resistance augmentation, but arteriolar and venular dilatation for the reduction of both peripheral pressure and flow partially opposes it.

3. Hematocrit reduction, due to the priming volume, decreases total resistance. Venular dilatation for $\mathrm{C}_{\mathrm{AO} 2}$ reduction (with the same $\mathrm{p}_{\mathrm{O} 2}$ ) and consequent arteriolar dilatation and interstice drying up amplify it.

4. Oxygen consumption reduction causes a district dependent venular constriction, less evident where metabolic activity is lower.

5. Proteinic concentration reduction, due to the priming volume, causes a strong transcapillary flow to interstice and capillary constriction with consequent reduction of flow. Venular dilatation prevails for small reductions and arteriolar pressure decreases with consequent arteriolar dilatation; vice versa for great decreases. It influences total arterial resistance (Fig.6).

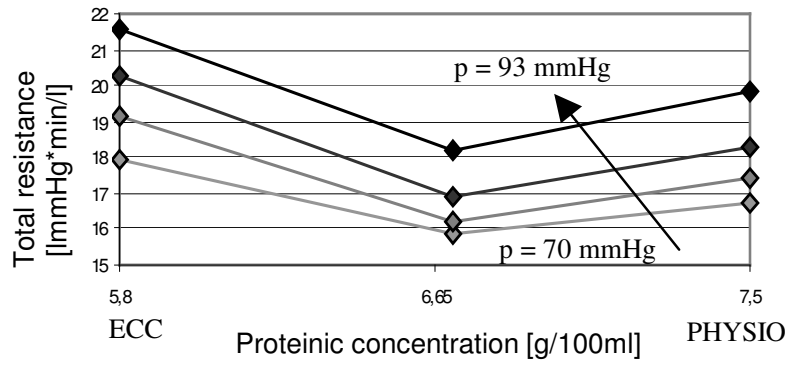

Fig.6: Total resistance depending on proteinic concentration and input pressure.
During simulated ECC accounting for all the alterations at once, the sum of these phenomena was observed. In particular there is flow reduction and venular dilatation, much more elevated where metabolic consumption reduction is higher and in the districts near the heart with an elevated pulsatility of flow in physiological conditions. There is also a reduction of capillary hydrostatic and oncotic pressure with high transcapillary flow of fluid and interstitial accumulation.

\section{Discussion and conclusions}

The described model can reproduce peripheral vasomotion with a deeper insight thus non limited by the classical black box description. So it can explain cardiovascular behaviour in non physiological conditions, like during ECC. This kind of modelling has some limits e. g. the lack of variability in regional configuration and control mechanisms, and the empirical modelling of controls. However, it is a first integration between whole arterial tree modelling and local transport and control mechanisms.

\section{References}

[1] Swanson WM, Clark RE. Cardiovascular System Simulation Requirements, Design and Performance. Journal of Bioengineering 1977;1:121-33

[2] Bergel DH. The Static Elastic Properties of the Arterial Wall. Juornal of Physiology 1961;150:445-47

[3] Burattini R, Gnudi G. Computers Identification of Models for the Arterial Tree Input Impedance: Comparison Between two new Models and First Experimental Results. Medical \& Biological Engineering \& Computing 1982;20:134-44

[4] Victorino GP, Newton CR, Curran B. Effect of Hypertonic Saline on Microvascular Permeability in the activate Endotelium. Journal of Surgical Research 2003;112:79-83

[5] Landis M, Pappenheimer JR. Excange of Substances through the Capillary Wall. American Physiological Society 1963:2,II,29:961-1034

[6] Groebe K. Precapillary Servo Control of Blood Pressure and Postcapillary Adjustment to Tissue Metabolic Status a New Paradigm for Local Perfusion Regulation. Circulation 1996;94:1876-85

[7] Singh MP, Sharan M, Aminataei A. A Matematical Model for the Computation of the Oxygen Dissociation Curve in Human Blood. Bio Systems 1989;22:249-60

[8] Davis MJ. Myogenic Response Gradient in Arteriolar Network. American Journal of Physiology 1993;264:216879

Address for correspondence:

Ing. Ettore Lanzarone, Prof. Maria Laura Costantino Department of Bioengineering

Politecnico di Milano

Piazza Leonardo da Vinci, 32

20133 Milano, Italy 